\title{
Ligand-induced Conformational Changes in ras p21: A Normal Mode and Energy Minimization Analysis
}

\author{
Jianpeng $\mathrm{Ma}^{1}$ and Martin Karplus ${ }^{1,2 *}$
}

\author{
${ }^{1}$ Department of Chemistry and \\ Chemical Biology, Harvard \\ University, 12 Oxford St \\ Cambridge, MA 02138, USA \\ ${ }^{2}$ Laboratoire de Chimie \\ Biophysique, Institut Le Bel \\ 4, rue Blaise Pascal \\ 67000, Strasbourg, France
}

\begin{abstract}
A normal mode and energy minimization of ras p21 is used to determine the flexibility of the protein and the origin of the conformational differences between GTP and GDP-bound forms. To preserve the integrity of the structures, a hydration shell of water molecules was included as part of the system. Certain low-frequency modes were found to have high involvement coefficients with the conformational transition between the GTP and GDP-bound structures; the involvement coefficients of some of the modes increase when the $\gamma$-phosphate group is removed. Two unstable modes that appear in the GTP-bound structure upon deletion of the $\gamma$-phosphate group were determined and shown to have dominant contributions in the regions of switch I and switch II; there was also a significant displacement of loop 1 . The initial motion in these regions is predicted by the modes to be approximately perpendicular to the direction of the transition from the GTP-bound state to the GDP-bound state. The overall conformational change in the switch I and II regions involves rearrangements of the protein backbone within these regions, rather than rigid body motion. Differences in the low-frequency modes of the GTP and GDP-bound forms appear to play a role in ligand binding. A coupling between the helix $\alpha 3$ position and the deletion of the $\gamma$-phosphate group may be involved in the interaction with GAP. The oncogenic mutation G12D leads to a global increase in the rigidity of the protein. Thus, the mutant is likely to have a higher barrier for the conformational change to the inactive form; this would slow the transition and could be related to its oncogenic properties.
\end{abstract}

(C) 1997 Academic Press Limited

Keywords: conformational change; energy minimization; normal mode analysis; computer simulation; point mutation ively. The transition from the active form to the inactive form occurs when ras p21 hydrolyzes the $\gamma$-phosphate group of the bound GTP substrate. The hydrolysis reaction is facilitated by the association between p21 and a protein called GAP (GTPase Activating Protein), although ras p21 alone possesses a weak intrinsic GTPase activity (Barbacid, 1987). It has been shown that point mutations of certain amino acid residues, particularly those in positions 12, 13 and 61, can lead to cancer (Barbacid, 1987). Although the ras system has been studied in great detail, the mechanism by which the mutants induce cancer is not fully understood at the molecular level. There is evidence to suggest that the increase in the lifetime of the active conformational state is important (Gibbs et al., 1984; Seeburg et al., 1984; Manne et al., 1985; Der et al., 1986), although other factors may be involved (Barbacid, 1987). If either the hydrolysis of the $\gamma$-phosphate group or the conformational 
change or both are inhibited, the "on" state is prolonged and can result in a continuation of the signal for cell growth (Barbacid, 1987).

One approach to obtain a better understanding of the coupling between GTP hydrolysis and the conformational change is to use computer simulations, which have been widely employed to study structure-function relations in biological systems (Brooks et al., 1988). Among the various simulation methods, a normal mode analysis is particularly useful for obtaining information about the vibrational motions and conformational changes of proteins. Early examples of such applications are the studies of hinge-bending motion of lysozyme (Brooks \& Karplus, 1985) and hexokinase (Harrison, 1984); recent examples are the study of two N-terminal domains of CD4 (Sanejouand, 1996), the conformational transition in ATCase (Thomas et al., 1996a,b) and the hinge-bending motion in citrate synthase (Marques \& Sanejouand, 1995). Since the normal mode calculation makes use of the harmonic approximation, cases where the structure undergoes a large conformational change and anharmonic effects (e.g. barriers) are important, are not described fully by such an analysis. Nevertheless, the normal mode results can be used to study the initial stages of the displacement so as to obtain insights into the origin of the conformational change. Of particular interest are unstable modes, modes with imaginary frequencies (Choi \& Straub, 1994; Stratt, 1995), that are introduced by a triggering event (here the hydrolysis of GTP). They give information about the motions that are expected to be induced by the conformational trigger.

Here, we calculate and examine the normal modes of several GTP and GDP-bound forms of ras p21. Since minimization of the structure is required prior to a normal mode analysis (Brooks et al., 1988), we first examine the structures obtained after minimization. Although minimization in vacuum leads to large deviations from the $X$-ray structures, we show that in the presence of a water shell the minimized structures are in good agreement with X-ray results. Given the importance of the water for the structural integrity, the normal modes are calculated for the protein with a water shell. We describe briefly the distribution of the normal mode amplitudes between the protein and the solvent. We then determine the nature and number of low-frequency (large-amplitude) modes in the GTP and GDP-bound forms that have a significant overlap with the conformational differences between the two, i.e. we isolate the normal modes that could contribute to the conformational change. To complement this analysis, we delete the $\gamma$-phosphate group in the GTP structure and determine the unstable modes that are introduced and evaluate the structural change expected from them. Since the water shell plays an important role in the integrity of the native structure, the motion of water molecules induced by the removal of the $\gamma$-phosphate group are also examined. The G12D mutant, which is the most malignant (cancer-inducing) mutation at this position (Seeburg et al., 1984) is studied to determine the differences in the normal modes and fluctuations introduced by the mutation. Equal-time correlation functions are calculated from the normal modes and used to examine the differences between the GTP and GDPbound forms. Finally, we summarize the conclusion concerning the conformational transition from the normal mode analysis and compare the results to those based on crystallographic data and molecular dynamics simulations.

\section{Results and Discussion}

We briefly describe the results of minimization with and without water and the water contribution to the normal modes. We then analyze the important features of the conformational change between the GTP and GDP-bound forms of ras p21 deduced from X-ray structures. The normal mode results are presented next and used to interpret some of the X-ray results.

\section{Overall characteristics of calculations}

\section{Structural results}

In the presence of the hydration shell consisting of explicit water molecules, the deviation of the protein structures upon full minimization (rms gradient $10^{-7}$, no negative mode) was found to be very small (see Table 1). The octahedral coordination geometry of the $\mathrm{Mg}^{2+}$ cation and the overall structure of the active site were dramatically stabilized, relative to the results without water. Without the hydration shell, much larger deviations were observed on minimization (see Table 1). We also determined the effect of the solvent shell on the conformational differences between the main-chain atoms of the GTP-bound form and the GDP-bound form. The differences between the results with and without minimization (the X-ray structures) are given in Figure 1 for the systems that do and do not have a hydration shell. The vacuum minimization can be seen to introduce significantly larger changes than those for the solvated structures, although the overall features of the conformational changes are preserved in the absence of water. These results provide evidence for the greater integrity of the structure obtained for ras p21 with the solvent shell. Consequently, the sol-

Table 1. The rms deviation $(\AA)$ from the minimizations with and without the hydration shell

\begin{tabular}{lcc}
\hline & Main chain & All atom \\
\hline WT GTP & $0.46(1.17)$ & $0.51(1.92)$ \\
WT GDP & $0.53(1.07)$ & $0.73(1.37)$ \\
G12D mutant & $0.51(0.98)$ & $0.64(1.32)$ \\
\hline
\end{tabular}

The data in parentheses are for the results without the water molecules. WT, wild-type. 


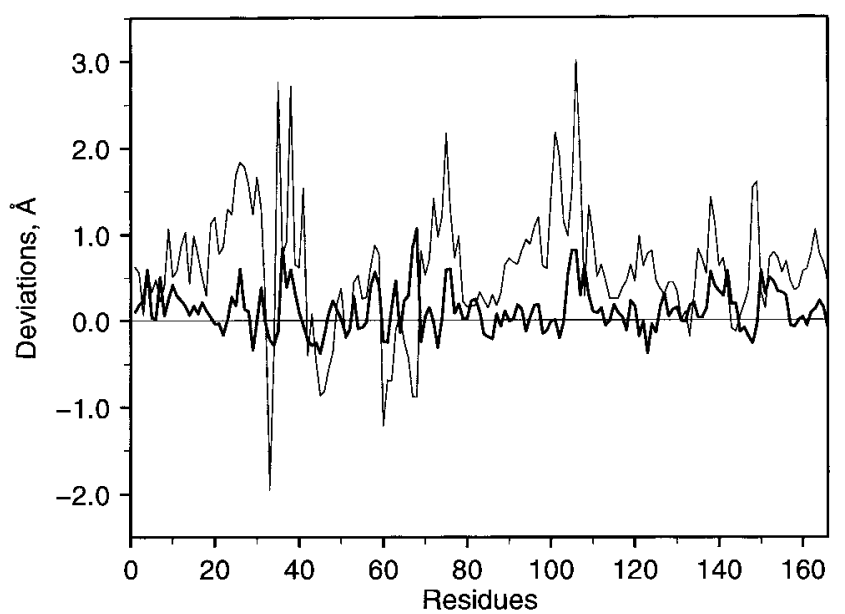

Figure 1. Deviation from the $\mathrm{X}$-ray results of the conformational difference between the main-chain atoms of the GTP-bound form and the GDP-bound form found after minimization. The thick line gives the results from the minimization with the hydration shell and the thin line corresponds to the vacuum minimization.

vated system normal mode results were used for interpreting the structural transition between the GTP and GDP-bound form of ras p21.

\section{Water contribution to normal modes}

Since a hydration shell composed of explicit flexible water molecules was included in the normal mode calculation, we examined the distribution of the modes between the water molecules and the protein. The average contribution of each mode to the atomic fluctuations of the protein, summed over all protein atoms, and of water, summed over all the water molecules, was calculated. Figure 2(a) shows a plot of the results for the first 1000 modes (from $6.11 \mathrm{~cm}^{-1}$ to $107.24 \mathrm{~cm}^{-1}$ ) for the wild-type CTP-bound form; an expanded plot of the first 200 modes (from $6.11 \mathrm{~cm}^{-1}$ to $40.77 \mathrm{~cm}^{-1}$ ) is given in the inset. The contributions to the atomic fluctuations from the protein atoms and the water molecules are very similar for all of the modes. For the first 850 modes, the water fluctuations are somewhat larger, even though there are more than twice as many protein atoms as water atoms; the two curves cross in the neighborhood of $850 \mathrm{~cm}^{-1}$. This provides evidence that the water motions are closely coupled to those of the protein; i.e. no "pure" water modes occur in the important lowfrequency range. Similar results were obtained for the other protein structures. Figure 2(b) gives the magnitude of the rms fluctuation for the wild-type GTP-bound structure calculated from the normal modes at $300 \mathrm{~K}$ (both with and without water) together with the fluctuation magnitudes derived from the $B$-factor obtained from X-ray measurement; the relation between the mean-square displacement of atom $i$ and the $B$-factor is given by $B=8 \pi^{2} / 3\left\langle\Delta r_{i}^{2}\right\rangle$. Although there is considerable cor-
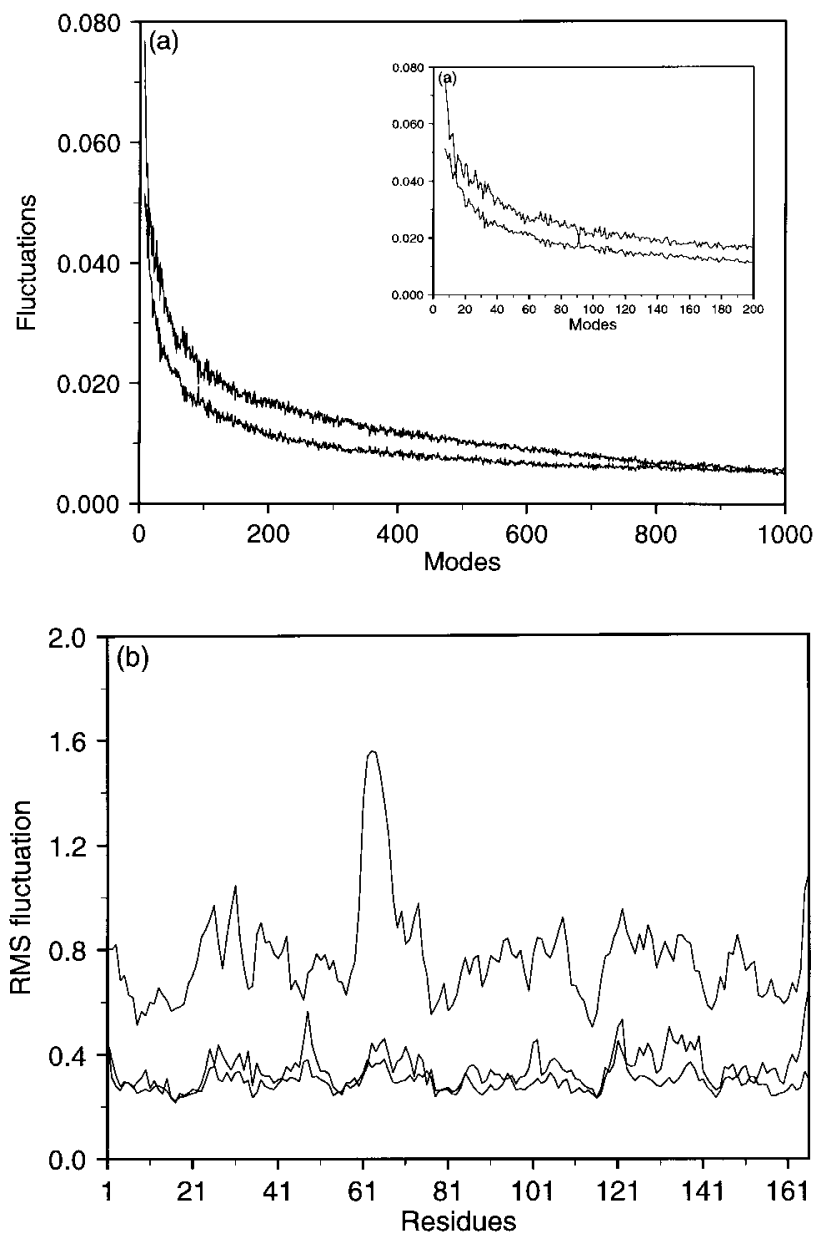

Figure 2. (a) The averaged contributions to the atomic fluctuations from the protein atoms and water molecules. Data are shown for the first 1000 modes calculated for the wild-type GTP-bound form. The upper curve is for the water molecules and the lower curve is for the protein. An amplified version for the first 200 modes is shown as an inset. (b) $\mathrm{C}^{\alpha}$ rms fluctuations as a function of residue number for the wild-type GTP structure at $300 \mathrm{~K}$. The upper curve gives the rms fluctuation derived from the $\mathrm{X}$-ray $B$-factors, the middle curve gives the results from the normal mode analysis without water, and the slightly lower curve gives the results from the normal mode analysis with water.

respondence in the relative values of the fluctuations, the calculated magnitudes are significantly smaller than those estimated from the $B$-factors, and the variation from one residue to another is less pronounced. The major difference is the absence in the calculated values of the large $B$-factor peak in the neighborhood of residue 61 (switch II region), which probably arises from disorder in the crystal. Also, some of the difference between the calculated and experimental fluctuations may be due to rigid rotation contributions to the crystal values (Kuriyan \& Weis, 1991).

The fluctuations from the modes including the water shell are only slightly smaller than the vacuum values. This result is different from that 
obtained by Teeter \& Case (1990), who found a greater reduction in the fluctuation for crambin with a water shell. However, they used a water shell of about $5 \AA$ thickness around the protein, which corresponds to many more water molecules than those in the single shell of "structural water molecules" used here, i.e. a $5 \AA$ shell in the case of ras p21 would correspond to about 1400 water molecules in contrast to the 400 in the present model system. Thus, it is not surprising that there was a greater reduction in the fluctuations observed by Teeter \& Case (1990), since a thick shell of water molecules may coat the protein with a layer of "ice" that rigidifies the structure. It should be noted that the use of a water shell in a molecular dynamics simulation of myoglobin yielded values of the atomic fluctuations very similar to those for a fully solvated system (Steinbach \& Brooks, 1993).

\section{Conformational changes from X-ray structures}

Representations of the structures of the wildtype active GTP-bound form of ras p21 and the corresponding inactive GDP-bound form are given in Figure 3(a); the legend lists the structural elements of the protein. The main structural features of the active site of the GTP-bound ras p21 are the octahedral coordination of the $\mathrm{Mg}^{2+}$ and the interactions between the $\gamma$-phosphate group and the loops located in the vicinity (Figure 3(b)). The six ligands of $\mathrm{Mg}^{2+}$ are provided by six oxygen atoms donated by the protein, nucleotide and two water molecules. They are the oxygen atoms of the $-\mathrm{OH}$ groups of the side-chain of Thr35 (loop 2) and Ser17 (helix $\alpha 1$ ), the oxygen atoms of the $\beta$ and $\gamma$ phosphate groups of the substrate and the oxygen atoms of two tightly bound water molecules. A molecular dynamics simulation of solvated structures (unpublished results) demonstrated that the octahedral geometry of the metal ion coordination is well preserved and plays an important role in maintaining the structure of the active site. The $\gamma$-phosphate group forms hydrogen bonds with the side-chain -OH and the main-chain -NH- of Thr35 (loop 2) and with the main-chain -NH- of Gly60 (loop 4). The hydrogen bond between the $\gamma$-phosphate group and Gly60 helps to keep loop 4 in the active conformation. The $\gamma$-phosphate group also contacts the side-chain of Lys16 (see Figure 3(c)) at the $C$ terminus of helix 1, next to loop 1. Loop 8 and loop 10 contact the guanine base. All five loops $(1,2,4,8$ and 10) that are involved in binding of the nucleotide are facing the cytosol when the protein is attached to the cell membrane (Wittinghofer \& Pai, 1991). Loop 2, which interacts with both $\mathrm{Mg}^{2+}$ and the $\gamma$-phosphate group, and loop 4, which interacts with the $\gamma$-phosphate group, which in turn interacts with $\mathrm{Mg}^{2+}$, form a tightly coupled system with the $\gamma$-phosphate group and $\mathrm{Mg}^{2+}$. Loop 2 and loop 4, both of which change their conformations between the GTP and GDP-bound forms (see below), have been identified as interaction sites for the GAP protein (Wittinghofer \& Pai, 1991).

The conformational differences between the GTP and GDP structures in the region of molecular switch II (mainly loop 4 and helix 2) are evident in Figure 3(a). Loop 4 moves away from the nucleotide in the GDP-bound form. This appears to be due in part to the loss of hydrogen bonding contact between the main-chain -NH- of Gly60 in loop 4 and the $\gamma$-phosphate group. Part of helix 2 (from Ala66 to Asp69) in the GTP-bound form unwinds in the GDP-bound form. This secondary structural change is believed to be important in the shift of loop 4 . The conformational difference in the region of molecular switch I (mainly loop 2, see Figure 3(a)) involves, in addition to the main-chain displacement, a large change in the direction of the side-chain of Tyr32 and smaller changes in the side-chains of Thr35 and Ile36. In the GTP-bound form, Tyr32 partially blocks the nucleotide-binding pocket and, in the GDP-bound form, it points in a different direction and forms a hydrogen bond with Tyr40. In the crystal structure of the $\mathrm{GppCH}_{2} \mathrm{p}$ complex, Tyr32 is directly bound to the $\gamma$-phosphate group (Milburn et al., 1990). The sidechain of Thr35 forms hydrogen bonds with both the $\gamma$-phosphate group and $\mathrm{Mg}^{2+}$ in the GTPbound form but points out to the solvent in the GDP-bound form. The displacement of Thr35 appears to be coupled to the motion of the sidechain of Ile36. The hydrophobic side-chain of the latter is in water in the GTP-bound active form, and it moves closer to the surface of the protein in the GDP-bound form (Wittinghofer \& Pai, 1991).

Figure 4(a) shows the conformational differences between the main-chain atoms of the wild-type GTP-bound form and the GDP-bound form as a function of residue number. The crystal structures were least-squares fit to each other in terms of the main-chain atoms; regions with large conformational differences were excluded in the fitting procedure (the following regions were included in the alignment of the two structures: residues 1 to 25,39 to 59,77 to 121 and 125 to 166 ). For the region of molecular switch $I$, the major structural differences in the main chain start at residue Val29 (Asp30 has a significantly different side-chain orientation) and end at Ser39 (Asp38 has a different side-chain orientation). The region of molecular switch II has conformational differences beginning at Ala59 and ending around Gly75; the side-chain of Tyr71 is differently oriented in the two forms. There are glycine residues at the two ends of switch II (Gly60 and Gly75, Gly77) which could serve as hinges for the conformational change (but see below). There are also significant structural differences in the region 41 to 52 involving $\beta$ strand 2 and loop 3, and around Arg123. The flexibility of this part of protein, which seems not to have been considered, is analyzed in terms of the normal modes below.

In Figure 4(b), we present the differences of the pseudo-dihedral angles of the GTP and GDP struc- 


\section{The GTP-bound form}

(a)

\section{The GDP-bound form}
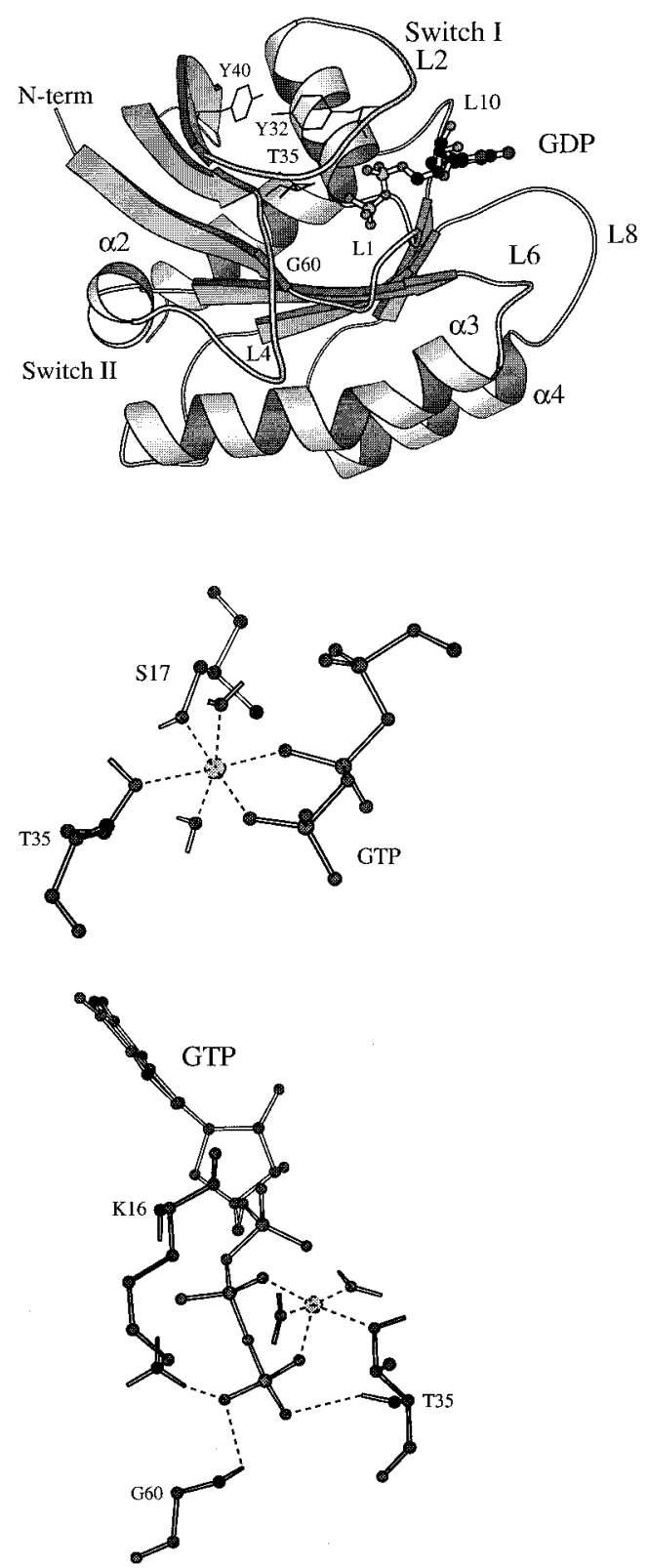

Figure 3. (a) Representations of the wild-type active GTP-bound form and the inactive GDP-bound form of ras p21. The nucleotide substrates are represented by a ball-and-stick model. The side-chains of Y32 and Y40 are shown to indicate an important conformational difference in the switch I region. The structure elements of ras p21 are marked. It consists of five $\alpha$-helices (they are $\alpha 1$ (residues 16 to 25), $\alpha 2$ (residues 65 to 74), $\alpha 3$ (residues 87 to 103 ), $\alpha 4$ (residues 127 to 137) and $\alpha 5$ (residues 152 to 164 )); a central $\beta$-sheet with six strands, five of which are antiparallel and one of which is parallel (they are $\beta 1$ (residues 1 to 0 ), $\beta 2$ (residues 38 to 46 ), $\beta 3$ (residues 50 to 58 ), $\beta 4$ (residues 77 to 84 ), $\beta 5$ (residues 110 to 117), $\beta 6$ (residues 140 to 144)); and ten loops (they are L1 (residues 10 to 15), L2 (residues 26 to 36 ), L3 (residues 47 to 48), L4 (residues 59 to 64), L5 (residues 75 to 76), L6 (residues 84 to 86), L7 (residues 104 to 110), L8 (residues 117 to 126), L9 (residues 138 to 140), L10 (residues 145 to 151)). The Figures were generated by the MOLSCRIPT program (Kraulis, 1991). (b) Stereo plot of the octahedral coordination of the magnesium ion in the GTPbound structure. The metal ion is represented by the black-and-white sphere; for the GTP substrate, only the phosphate groups are shown, and for the amino acid residues, the heavy atoms and polar hydrogen atoms are shown. (c) Stereo plot of the hydrogen bonding interactions of the $\gamma$-phosphate group, part of the metal ion coordination is also indicated, and for amino acid residues, only the heavy atoms and polar hydrogen atoms are shown.

tures as a function of residue number. The pseudodihedral angle $i$ is defined as the dihedral angle between atoms $i-1, i, i+1, i+2$. It is useful in analyzing conformational changes because the individual $\Phi$ and $\Psi$ angles can have large anticorrelated changes (McCammon et al., 1977) that 

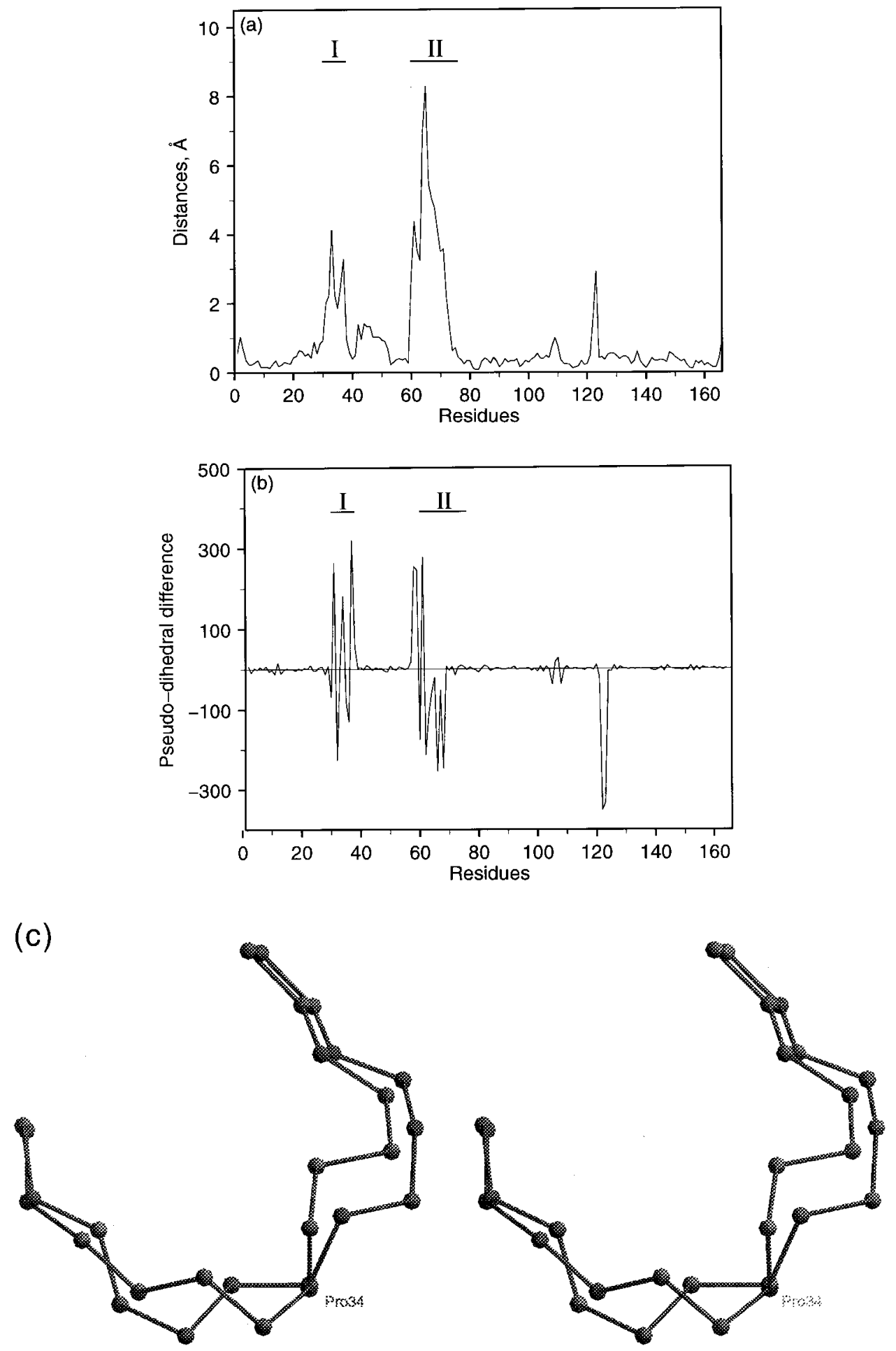

Figure 4. (a) Conformational differences (in $\AA$ ) between the main-chain atoms of the wild-type GTP-bound form and the GDP-bound form. High peaks in the regions of the molecular switch I (residues 30 to 38) and switch II (residues 60 to 76) indicate the largest conformational changes. In addition, there are non-negligible changes for residues 41 to 57 and around Arg123. The two switch regions are marked. (b) The difference of the pseudo-dihedral between the wild-type GTP-bound form and the GDP-bound form. The difference data are plotted with respect to the index of the second atom " $i$ " (see the definition of the pseudo-dihedral in the text). The two switch regions are marked. (c) Stereo plot for the $\mathrm{C}^{\alpha}$ trace of the conformation of loop 2, the molecular switch I region. The active and the inactive structures were least-squares fit to each other with the same procedure as in (a).

leave the main-chain conformation essentially unchanged. An example of the utility of the pseudodihedral angles is found in the analysis of the rigid "lid" closure of the active site in triosephosphate isomerase (Joseph et al., 1990). Unlike that conformational change, loops 2 and 4 of ras p21 change 
their conformations primarily by internal "twisting" rearrangements, as indicated by the complex features of the pseudo-dihedral angles within the two switch regions (see Figure 4(b)). In the switch II region, the pseudo-dihedral angle difference ends at residue Gln70, while the conformational difference extends to Gly77. Since residues Gln70 to Thr74 are $\alpha$-helical $(\alpha 2)$ in both the GTP-bound and the GDP-bound form, this common structural feature moves rigidly. Residues Ser65 to Asp69 form an additional helical turn in $\alpha 2$ in the GTPbound form and it is part of the extended coil structure in the GDP-bound form, as can be seen clearly in the behavior of the pseudo-dihedral angles; this difference is crucial for accommodating the overall conformational change of switch II (Ma \& Karplus, 1997). While there is a large change in the pseudo-dihedral angles involving Gly60, there is almost none for Gly75 and Gly77. This raises doubts about the role of the latter as a hinge (compare for example with the results of the TIM (Joseph et al., 1990)). Moreover, with the large internal conformational changes that contribute to the displacement of switch II, a hinge appears not to be required. A similar conclusion applies to switch I, where there are no glycine residues in the appropriate positions.

The switch I region contains Pro34, which is highly conserved across different species (Lowy \& Willumsen, 1993). Although located in the middle of the conformational transition region, the $C^{\alpha}$ atom remains at approximately the same position upon overall alignment of the two structures and it appears to divide the displacement into two parts (see Figure 4(c)). There are some backbone displacement of the other proline atoms, although there is a dip at position 34 in Figure 4(a). The role of Pro34 in the conformational transition is of particular interest because key residues like Thr35 and Tyr32 are very close to it, as recently pointed out by Kjeldgaard et al. (1996). It is likely that the subdivision of the conformational transition region facilitates the specific reorientation of certain residues, such as Tyr32 (see below). Studies of the effect of the P34A mutation would be of interest in this regard.

Although the overall structure of the G12D mutant with GTP bound is very similar to that of the wild-type protein (Franken et al., 1993), evidence based on fluorescent intensity changes (Neal et al., 1990) suggests that the mutant does not undergo the conformational change observed in the wild-type protein when the $\gamma$-phosphate group is hydrolyzed. The Asp12 side-chain forms hydrogen bonds with the side-chain of Gln61 (NE2(61)OD1(12), $3.14 \AA$ ) and the protonated $\mathrm{OH}$ of the $\gamma$-phosphate group. The X-ray temperature factors for loop 4 are somewhat smaller in the mutant structure than in the wild-type (Franken et al., 1993). This suggests that the conformation of loop 4 in the mutant is stabilized by the extra hydrogen bond between Asp12 and Gln61. The greater rigid- ity of the G12D structure is demonstrated from the normal mode results (see below).

\section{Normal mode results}

\section{Mode involvement coefficients}

The GTP* form (the GTP-bound X-ray structure with the $\gamma$-phosphate group removed) serves as a starting point for studying the conformational transition from the active GTP-bound form to the inactive GDP-bound form. The involvement coefficients, defined in equation (5) in Methods, of the first 3000 modes of the GTP* form in the direction of the conformational transition are shown in Figure 5(a); the modes cover a frequency range from 0 to $368.55 \mathrm{~cm}^{-1}$. Modes with higher frequencies do not make significant contributions to the larger-scale vibrational motions because the overall rms amplitude varies as $1 / \omega$ (see equation (2)). The involvement coefficients were computed for the atoms on protein backbone plus the $C^{\beta}$ atom if a residue was not glycine. Including $\mathrm{C}^{\beta}$ is a simplified way of indicating a change in orientation of the side-chain. Most of the modes with the highest involvement coefficients are located in the low-frequency region. Figure 5(b) shows the involvement coefficients for the modes in the region from 0 to $48.0 \mathrm{~cm}^{-1}$. There are 16 modes with involvement coefficients larger than 0.12 . For comparison, we calculated the involvements of the modes in the wild-type GTP-bound form; the data are given in Figure 5(c). Comparing the results for the two structures shows that the two are relatively similar but there are several modes (around mode 40 with frequencies around $19 \mathrm{~cm}^{-1}$ ) that have significantly increased involvement coefficients in the GTP* form. This is one indication that the removal of the $\gamma$-phosphate group increases the potential for the protein to undergo the observed conformational transition.

Although there are modes with relatively high involvement coefficients, none of them is localized in the switch I or the switch II region. Since the vibrational modes are limited to internal motions, they do not change the position of the center-ofmass nor introduce any rotation. Thus, the motion in the regions of switch I and II must be coupled to motions in other regions. This makes it difficult to obtain detailed information concerning the nature of the motion from the involvement coefficients. However, it suggests that appropriate linear combination of those modes can lead to significant conformational changes in the regions of interest.

\section{Unstable modes in the GTP* form}

To obtain a clearer picture of the effect of the deletion of the $\gamma$-phosphate group in the GTPbound structure, we examined the unstable modes (modes with negative eigenvalues and imaginary frequencies) that appeared in the normal mode spectrum of the GTP* form. The existence of such 

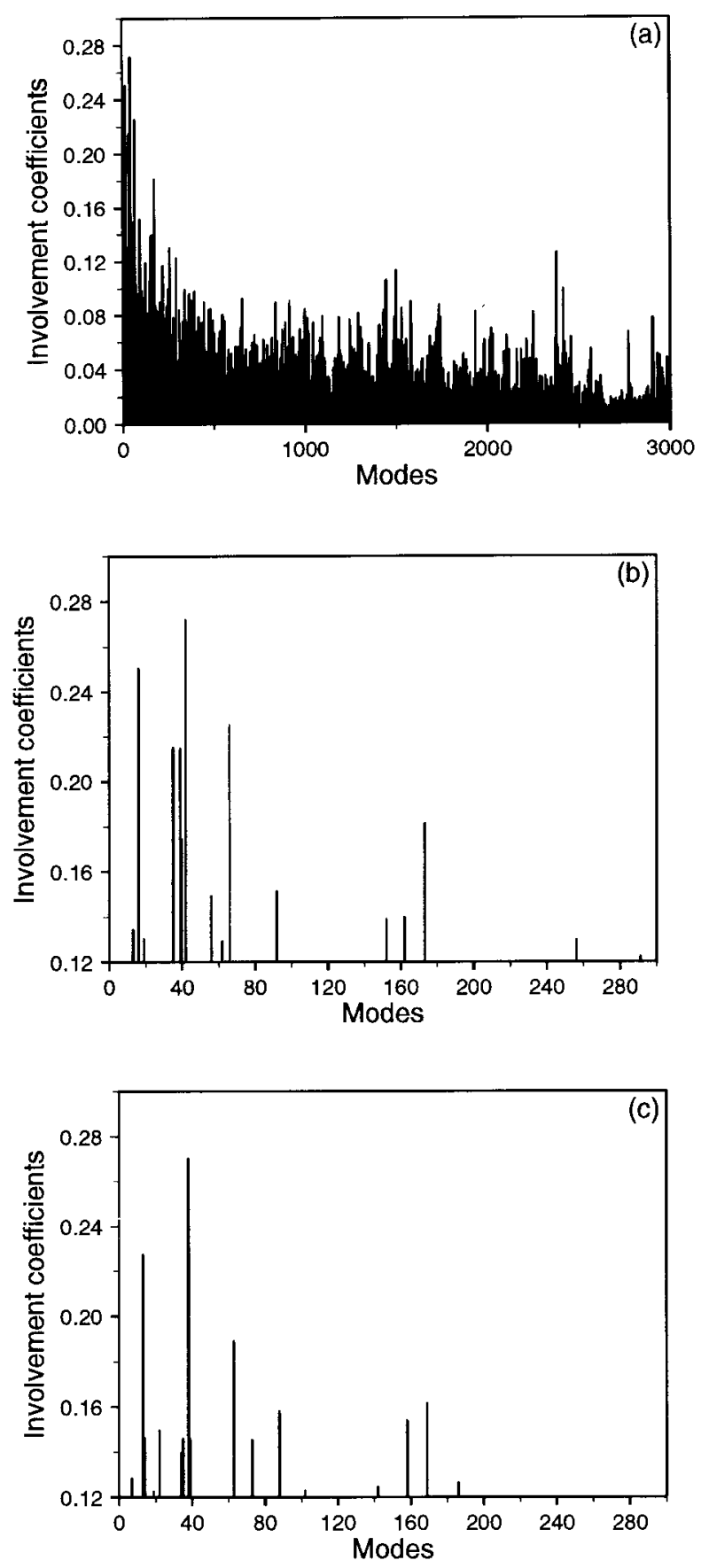

Figure 5. (a) The involvement coefficients (see equation (5)) of the first 3000 modes of the GTP* form. (b) Modes with a high involvement coefficient in the frequency region from 0.0 to $48.0 \mathrm{~cm}^{-1}$; all 16 modes with an involvement coefficient larger than 0.12 are in this region. (c) High involvement modes for the wild-type GTP-bound form.

negative eigenvalues indicates that the modified structure is no longer at an energy minimum. The motion along the unstable modes gives the initial direction of the expected conformational change. Unstable modes have been used in the studies of the dynamics of simple liquids (Stratt, 1995) and barrier crossing (Choi \& Straub, 1994).
For GTP*, there are two unstable modes with the frequencies -49.41 and $-25.38 \mathrm{~cm}^{-1}$. To determine the simultaneous effect of the unstable modes, we performed steepest descent minimization in the absence of the $\gamma$-phosphate group. The displacements of the structure as a function of residue number are shown in Figure 6(a). Three regions show the largest displacements. Of these, the two molecular switch regions have the largest change. The other region with significant displacements is loop 1 (residues 10 to 20), which does not have a significant conformational change between the GTP-bound and GDP-bound form in the crystal structure (see Figure 4). The influence of the deletion of the $\gamma$-phosphate group on this region is discussed further below. Other regions like helix 3, loop 8, and loop 10, all of which are close to the nucleotide (see Figure 3(a)), are also affected although the displacements are significantly smaller.

Since the structural water molecules are involved in stabilizing the GTP structure, it is of interest to observe how they respond to the deletion of the $\gamma$-phosphate group on minimization. This is shown in the stereo diagram in Figure 6(b). It is evident that water motions are highly non-uniform and that most of them are associated with the large structural changes in the protein (the loops in the switch I and switch II regions). Thus, the structural water molecules included in the system move essentially as an integral part of the protein, as expected from the normal mode analysis. To further test the validity of the resent results, we have minimized the vacuum GTP* system (no water) and the solvated GTP* system with two optimized water molecules replacing the $\gamma$-phosphate group. The results for the distribution and amplitudes of the displacements are essentially identical with those shown in Figure 6(a). This confirms that the motions observed are the intrinsic response of the protein to the deletion of the $\gamma$-phosphate group.

We also examined the unstable modes of the GTP-bound G12D mutant structure after the removal of the $\gamma$-phosphate group (i.e. the G12D* form). There is only a single negative mode; its frequency is $-37.80 \mathrm{~cm}^{-1}$. The magnitudes of the displacements from the minimization in the two molecular switch regions for the mutant are significantly smaller than those of the wild-type, as is indicated by the broken line in Figure 6(a). This is in accord with the fact that the structure of the G12D mutant, especially the two switch regions, has smaller inherent fluctuations than the wild-type protein, as already mentioned. In other words, it appears that the G12D mutant is more resistant to a conformational transition upon the removal of the $\gamma$-phosphate group than the wildtype. The large change in the loop 1 region for the G12D mutant is also expected, since Asp12 makes a hydrogen bond with the $\gamma$-phosphate group. 
The involvement coefficients for the unstable modes in the direction of the conformational transition are small, although the displacements due to the unstable modes are primarily in the regions that undergo conformational change (see Figure 6(a)); the involvement coefficients are 0.04 and 0.08 for the two negative modes in the GTP* form. This suggests that the initial structural change is not in the same direction as the overall transition. Since the conformational changes are each localized between two distinct points, this is expected if the transition involves a rotation of a loop from its initial to its final position. In this case, the initial displacement would be approximately perpendicular to the overall displacement (see Figure 6(c)). Of course, this is a highly oversimplifed description, since the actual conformational transition is much more complex, i.e. the conformational changes of ras p21 do not correspond to localized rigid body motions (see above).

The directions of some of the atomic displacement upon the minimization of GTP* form are shown in Figure 6(d). For clarity, only the $C^{\alpha}$ atoms in the loops around the active site are presented. As can be seen from the plots, the pep- tide segment 31 to 33 , especially the $C^{\alpha}$ atom of Tyr32, moves in a direction that points roughly downward towards the nucleotide, while the direction of motion of the $\mathrm{C}^{\alpha}$ atoms of residues 34 and 35, as well as the smaller displacements of residue 36 through 39, are in the opposite direction. This is in accord with the observation that Pro34 divides the motion of loop 2 into two distinct parts. Although there is no displacement of the $\mathrm{C}^{\alpha}$ of Pro34 between the GTP and GDP structure (see Figure 4(c)), there is significant initial motion, as shown in Figure 6(d). This makes it clear that the nature of the transition cannot be determined simply from the X-ray structure data for the limiting structures. The lower panel of Figure 6(d) gives the directions of the displacement of the Tyr32 side-chain. Its motion is consistent with its $\mathrm{C}^{\alpha}$, but it has even larger displacements in the same direction. The direction of this initial movement is critical to the conformational change of loop 2 (Ma \& Karplus, 1997). It is also of interest that Gly60 in the switch II region moves in an upward direction, while its two nearest neighbors move horizontally away from the nucleotide.

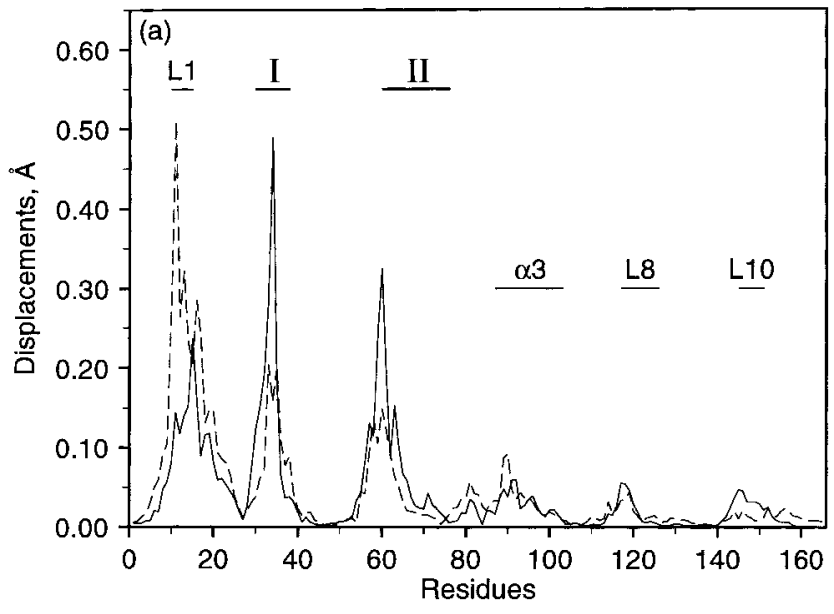

(b)

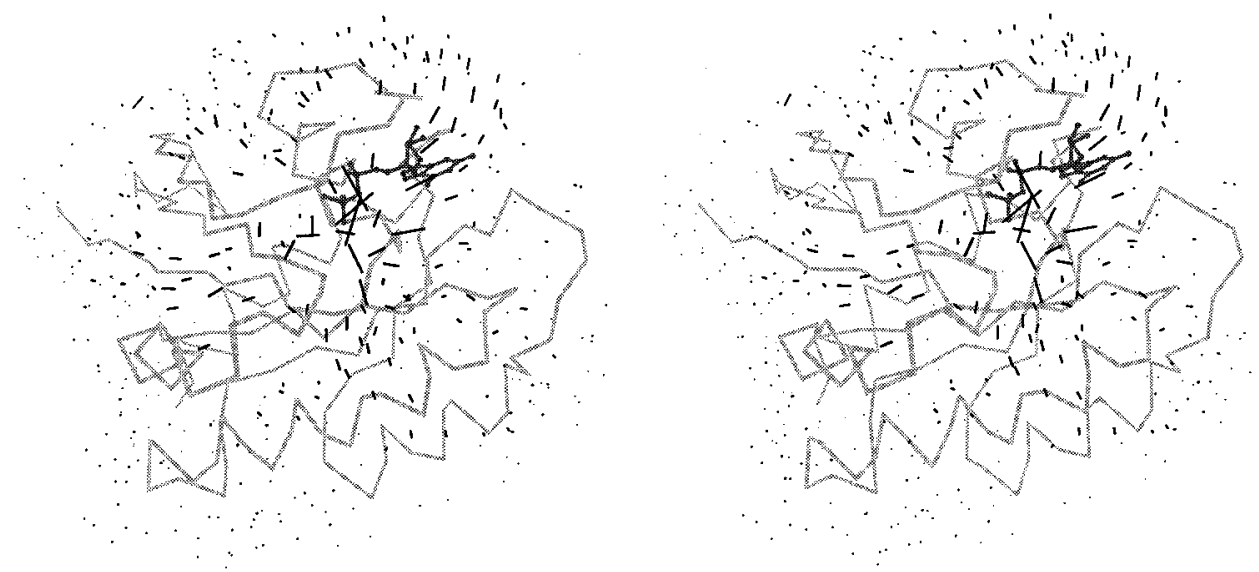

Figure $6(\mathrm{a}-\mathrm{b})$ (legend opposite) 


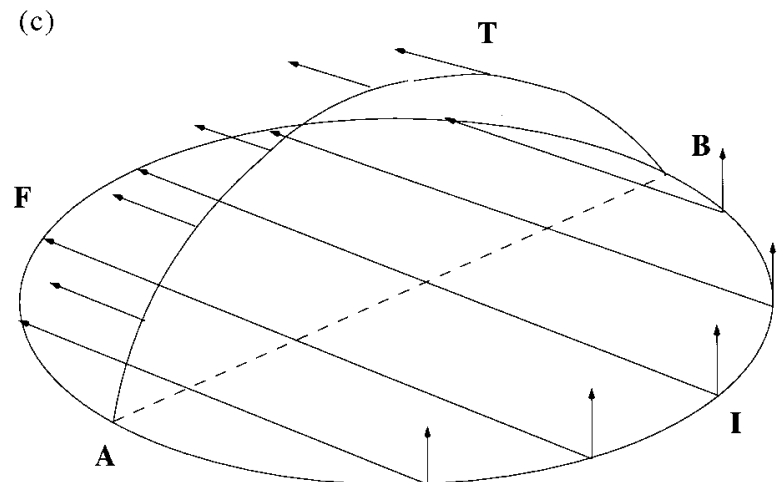

(d)
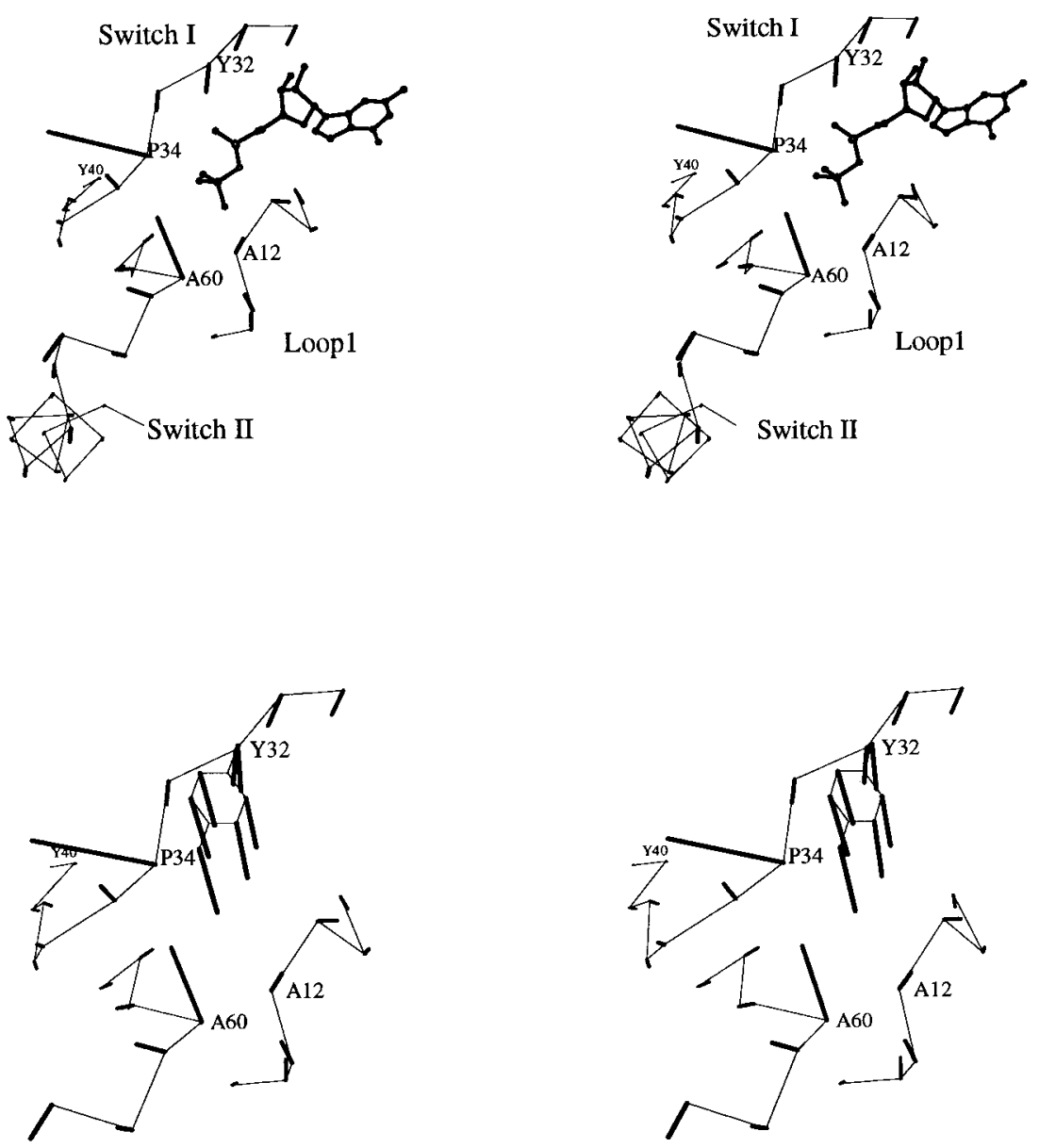

Figure 6. (a) The residue averaged atomic displacements resulting from steepest descent minimizations for the GTP* form (continuous line) and the G12D* form (broken line). Some important secondary structural elements and the locations of the two switch regions are marked. (b) Stereo view of motion of water molecules from the steepest descent minimization for the GTP* form. The protein $\left(\mathrm{C}^{\alpha}\right.$ atoms) is shown as a gray line and the dark line segments correspond to the displacements of water oxygen atoms. The GDP nucleotide is represented in ball-and-stick form. (c) A schematic representation of the direction of initial displacement for rotation of a loop. Loop A-B undergoes a conformational change; arc $\mathbf{I}$ represents the initial state and $\mathbf{F}$ is the final state. $\mathbf{T}$ represents an intermediate state. The short arrows on $\mathbf{I}$ and $\mathbf{T}$ represent the directions of the normal vectors, while the overall conformational difference vector is given by the arrows from I to F. (d) Upper panel: stereo plot of the atomic displacements upon minimization of the GTP* structure. Only the $\mathrm{C}^{\alpha}$ atoms in the active site region are shown; key residues are marked. The nucleotide substrate (in ball-and-stick representation) is included in the upper panel to indicate the binding site. Lower panel: stereo plot of the same region indicating the motion of the side-chain of Tyr32. 


\section{Equal-time cross-correlation functions}

The equal-time cross-correlations of the atomic fluctuations (equation (3)) describe the relations between the displacements of different parts of the protein. We computed the cross-correlation from the normal modes of the wild-type GTP-bound and GDP-bound form. As expected, the overall cross-correlations, which reflect the secondary and tertiary structure, are very similar in the two molecules. However, there are some significant differences due to the changes in the nature of the lowfrequency modes.

Figure 7 shows the cross-correlations for residues 1 to 71, which are mainly around the active site and include switch regions I and II. For the wild-type GTP-bound form (see Figure 7(a)), an "anchor-like" partly yellow region (residues 21 to 60 ) reflects the relatively high positive correlations
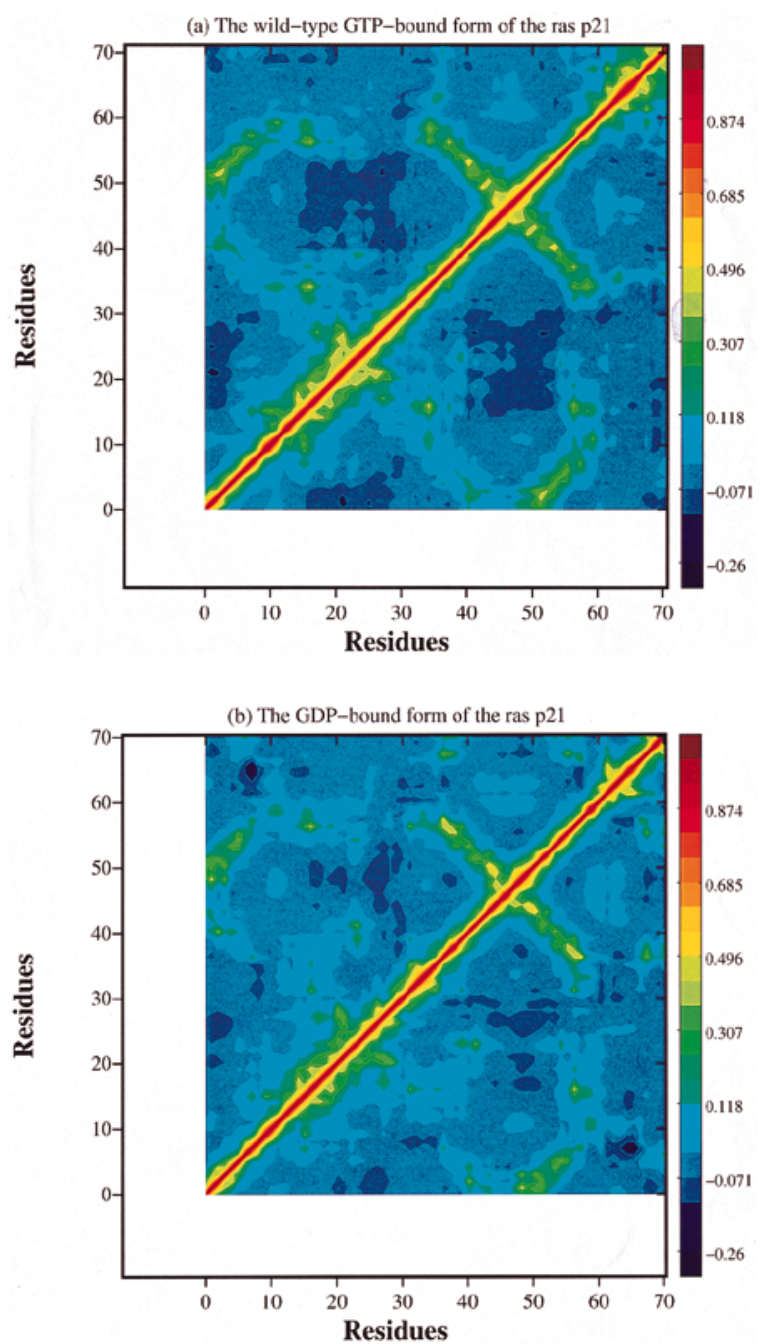

Figure 7. Equal-tune cross-correlaton functions of (a) the wild-type GTP-bound form and (b) the GDP-bound form. Each point on the map represents the correlation between a particular pair of residues (see the text). The color code indicates the magnitude of the correlation. Results are given for residues 1 to 71 . of the loops in the neighborhood of the $\gamma$-phosphate group. This anchor is weaker (in the region from residues 30 to 35) on the map of the GDPbound form (see Figure $7(b)$ ), i.e. the positive correlations between the motions of the residues in loop 1 and loop 2 are significantly reduced. There is also a global increase in the negative correlations for the GDP-bound form relative to the GTPbound form (not shown).

One aspect in the differences of the correlations in the vibrational motions of the GTP and GDPbound forms may be important in the function of ras $\mathrm{p} 21$. When the protein is in the active GTPbound form, the GTP substrate should be tightly bound and positioned to achieve the hydrolysis of the $\gamma$-phosphate group. However, in the inactive GDP-bound form, the protein needs to release the GDP product so that a new GTP molecule can be bound and the structure can return to the active form (Barbacid, 1987). Such an "unloading" and "reloading" process could be aided by the motions of the GDP-bound form. In fact, the higher negative correlations between the different parts of the protein, especially in the vicinity of the nucleotide, suggest that these motions contribute to opening and closing the active site. Further, in the GTPbound form, certain parts of the structure undergo more positively correlated motions, which could be important for the tight binding of the substrate. Figure 8(a) presents stereo plots for the vibrational patterns of modes 7 and 12 in the GTP-bound and the GDP-bound forms. The motions of the atoms in the loops that participate in the binding of the nucleotide have a more synchronized pattern in the GTP-bound form than in the GDP-bound form. In mode 7 , the regions with the largest vibrational amplitude in the GTP-bound form include the loop 2 and loop 8 regions that contact the guanine base. In the GDP-bound structure, mode 7 has its largest amplitude in regions away from the substratebinding site. Mode 12 in Figure 8(b) shows large amplitudes for both the GTP and GDP-bound forms. However, in the regions of the binding site, the motions of the atoms in loops are approximately in phase in the GTP-bound form and out of phase in the GDP-bound form. The other low-frequency modes (8 to 12 ) have corresponding characteristics.

In the GTP-bound form (see Figure 3(a)) Tyr32 lies across the nucleotide-binding pocket and limits the accessibility of the active site. In the GDPbound form, Tyr32 forms a hydrogen bond with Tyr40 and is oriented in the opposite direction, which results in a more open nucleotide-binding site. The change in the position of the side-chain of Tyr32 and its resulting interactions are likely to contribute to the changes in the correlations of the motions.

The same correlation functions were computed for the GTP-bound G12D mutant structure (not shown). Overall, the correlation map is very similar to that of the wild-type. However, the correlations between certain regions are observed to be 
increased. In going from the wild-type to mutant structure, the correlation coefficients between residue 12 and residues 31 and 61 were increased by about 0.23 and 0.29 , respectively, indicating that the motions in these critical regions are more correlated in the latter than in the former. This is due to the hydrogen bond between Asp12 and Gln61 in the mutant. The increased positive correlations among the residues around the $\gamma$-phosphate group in the G12D mutant is in accord with the conclusion that the mutant structure is inherently more resistant to conformational change than the wild-type.

\section{Atomic fluctuations}

To examine the overall influence of the $\gamma$-phosphate group on the vibrational dynamics, we computed the differences in the atomic fluctuations before and after the removal of the $\gamma$-phosphate group for the GTP-bound structures. The fluctu- ations, which were computed from the stable modes, reflect the global features of the influence of the deletion of the $\gamma$-phosphate group on the vibrations. Both the wild-type and the G12D mutant were studied. The results are shown in Figure 9. For the wild-type, significant increases of fluctuations are found in the two switch regions and the loop 1 region. However, the location of the peaks in Figure 9 in the region of the switch II are shifted in comparison with the conformational difference (see Figure 4(a)). Two other regions ( $\alpha 3$ and loop 8), which are located spatially close to the nucleotide-binding sites, also have an increase in their fluctuations. Thus, the removal of the $\gamma$-phosphate group introduces additional structural flexibility in certain key regions of the polypeptide chain, which enhances the possibility for the largescale conformational transition. In the G12D mutant, the influence of the removal of the $\gamma$-phosphate group on the structural flexibility is significantly different. The increases of the fluctuations at (a)
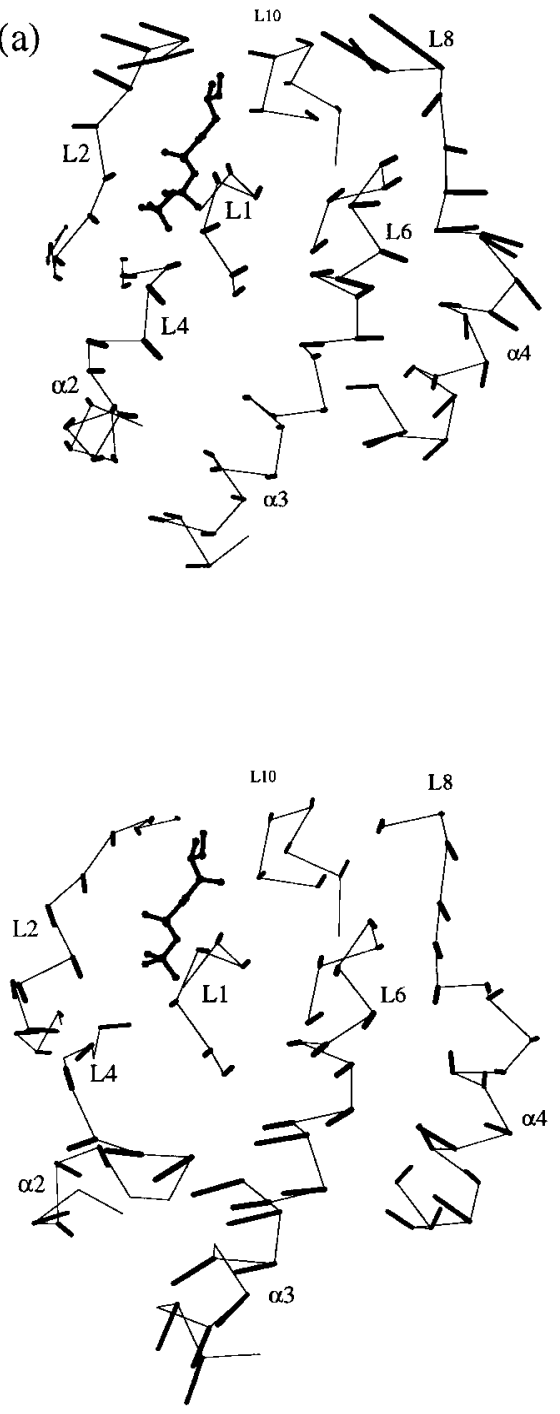
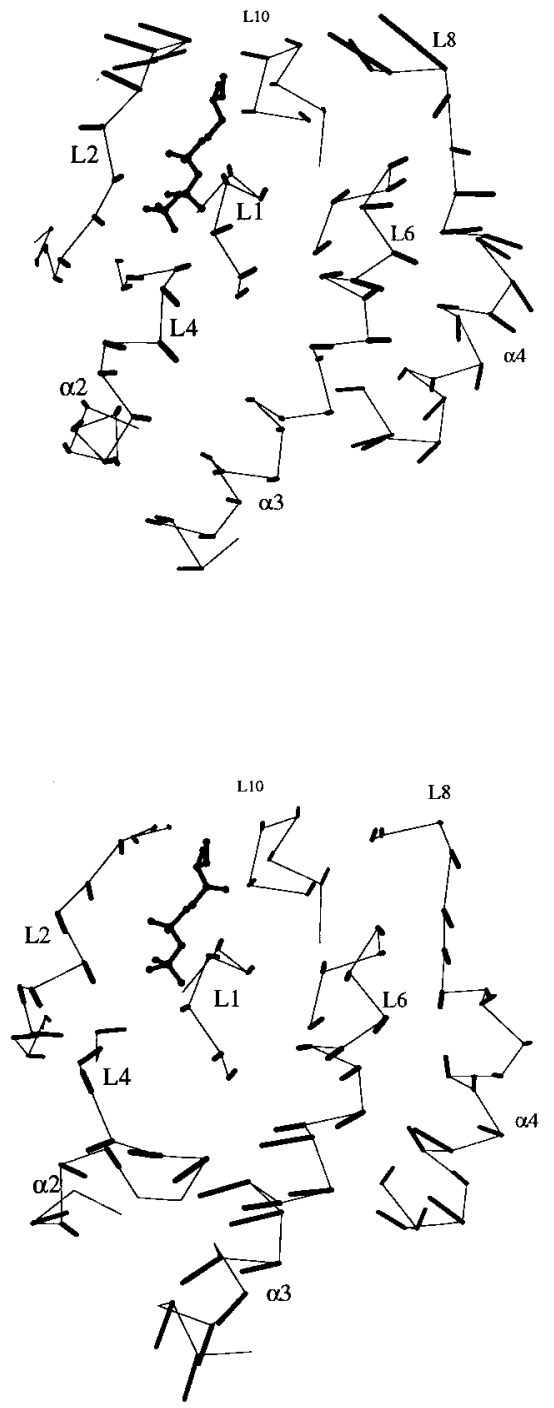

Figure 8(a) (legend on page 126) 
(b)
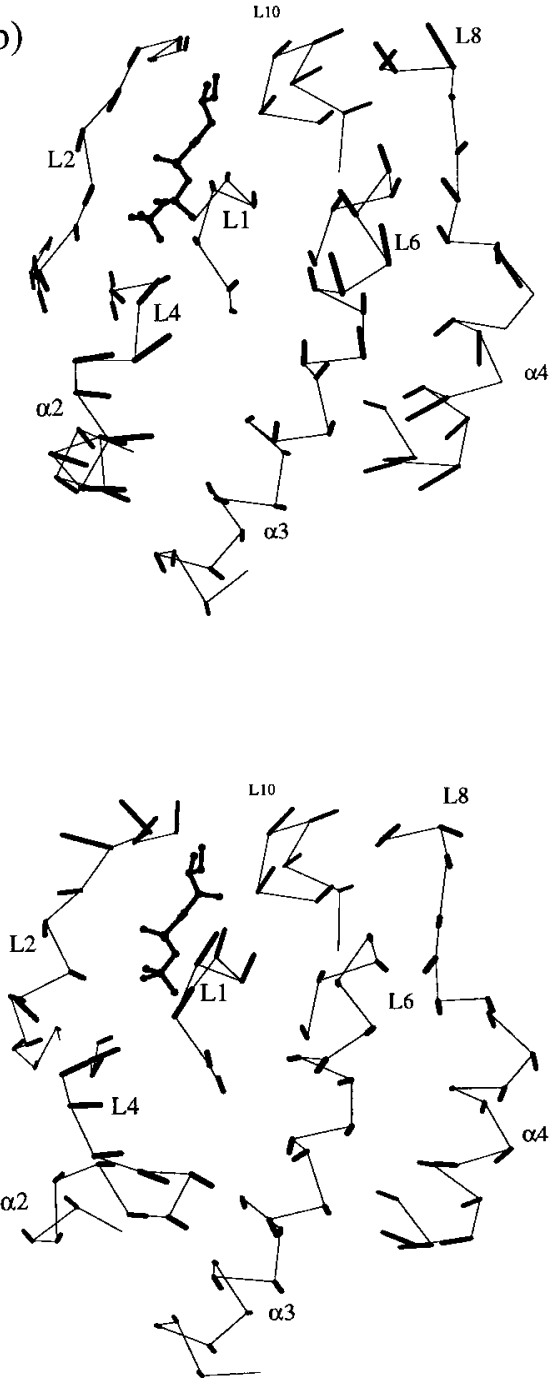
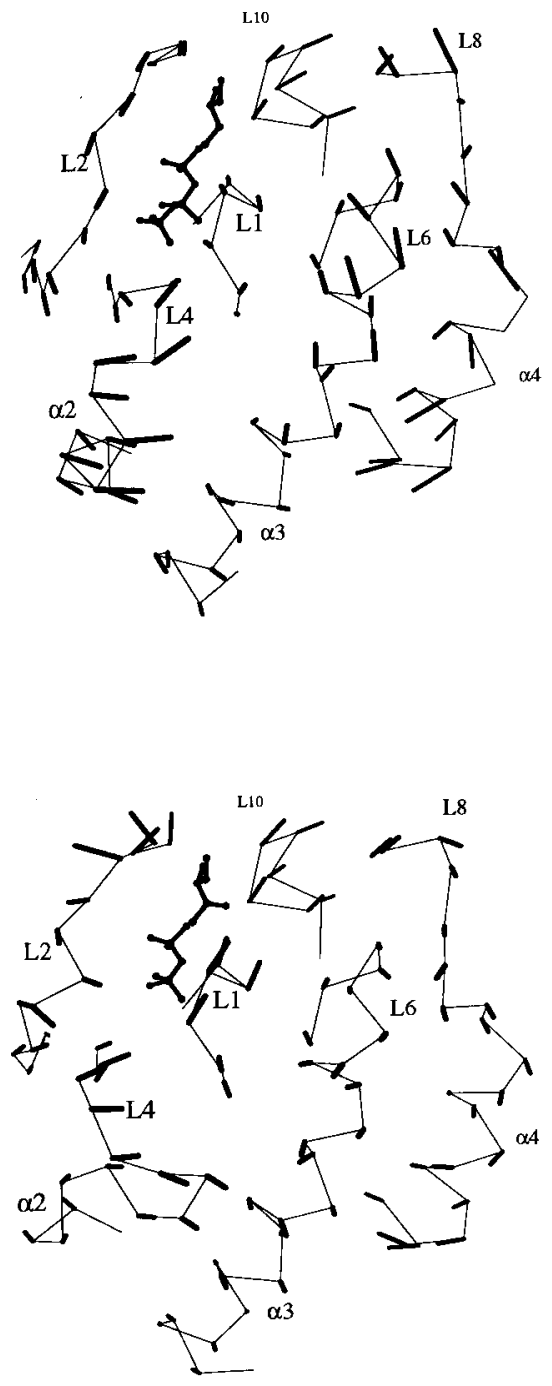

Figure 8. Stereo plots of (a) mode 7 and (b) mode 12. In both (a) and (b), the upper half is for the wild-type GTPbound form and the lower half is for the GDP-bound form. For clarity, only part of the molecule is shown. The $\mathrm{C}^{\alpha}$ atom and the substrate are represented. The magnitude of the motion of each $\mathrm{C}^{\alpha}$ indicated by the length of the heavy line was amplified ten times relative to that corresponding to that at $300 \mathrm{~K}$.

the switch II and loop 1 regions are much smaller. The flexibilities of two other regions $(\alpha 2$ and $\alpha 4)$ are decreased in the G12D mutant on removal of the $\gamma$-phosphate group.

\section{Concluding Discussion}

The conformational transition that occurs in ras p21 when the $\gamma$-phosphate group of the GTPbound state is hydrolyzed, is thought to play an essential role in the control mechanism; i.e. the GTP-bound form is active while the GDP-bound form is inactive. It is important to understand the nature of the transition both for its fundamental interest and as a possible target for drug design. To examine the initial steps of the transition, we have done normal mode and energy minimization analyses of a number of different forms of ras p21.
A hydration shell surrounding the protein was included to obtain a minimized structure for the normal mode analysis that is close to the X-ray structure. The water shell dramatically improves the integrity of the structure for energy minimization; i.e. the main-chain deviations of the minimized structure from the X-ray structure for the wild-type GTP-bound form with and without water molecules are 0.46 and $1.17 \AA$, respectively. In the modes of the solvated structure, there is an essentially equal contribution of protein and water motions. This demonstrates that the water shell is an integral part of the protein structure.

The normal modes of the wild-type GTP-bound form, which have a high involvement coefficient in the direction of the conformational transition, lie in the very low frequency region (below $48.0 \mathrm{~cm}^{-1}$ ). The removal of the $\gamma$-phosphate group significantly increases the involvement of some of these modes. 


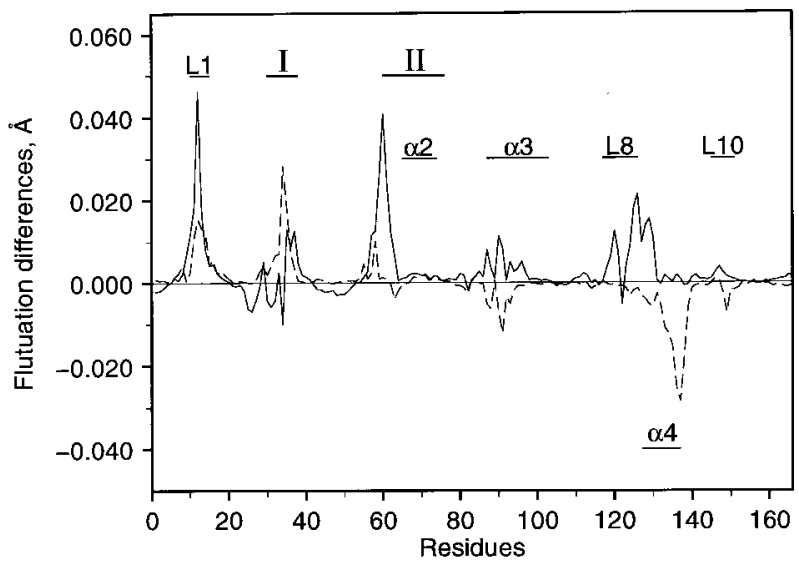

Figure 9. Differences in the atomic fluctuations calculated from the normal modes at $300 \mathrm{~K}$ as a function of residue number upon the removal of the $\gamma$-phosphate group. The continuous line gives the results for the wild-type GTP-bound form and the broken line gives the results for the G12D mutant. The locations of the two switch regions and important secondary structural elements are marked.

Two unstable modes are introduced in the GTPbound structure when the $\gamma$-phosphate group is removed. The motion due to both of them, as examined by minimization, leads to a large displacement of the switch regions. This provides direct evidence that the conformational changes of ras p21 are triggered by the leaving of the $\gamma$-phosphate group after hydrolysis. The motions of structural water molecules are found to be associated with those of the protein. The initial displacement in the switch regions is oriented essentially perpendicular to the actual displacement observed in going from the GTP to the GDP crystal structures, suggesting that a rotation is involved. However, the overall displacement is not a rigid body motion, unlike the lid motion in triosephosphate isomerase (Joseph et al., 1990). Instead, the transition involved internal dihedral angle rearrangements in both the switch I and switch II regions. The present analysis complements the results from molecular dynamics simulations (Foley et al., 1992; Díaz et al., 1995).

Analysis of the equal-time cross-correlation functions and the nature of the low-frequency modes for the GTP and GDP-bound forms indicates that the structure of the latter leads to opening and closing motion in the active-site region, while in the former there is a more synchronous motion that may facilitate tight binding of the nucleotide. In the cell, the exchange of GDP with GTP is achieved through the binding of the guanine nucleotide exchange factor (GEF; Feig, 1994). It has been shown (Howe \& Marshell, 1993) that this protein interacts with ras p21 in the $\alpha 2$ region. The significant shift in the motion of the $\alpha 2$ region in the GDP-bound form (see Figure 8(a)) may indicate that flexibility in that region plays a role in recruiting the exchange factor. However, the detailed mechanism of nucleotide exchange will be understood fully only after the structure of a complex of ras p21 and the exchange factor becomes available.

An interesting observation is the coupling between the release of the $\gamma$-phosphate group and the motion of helix $\alpha 3$. This can be seen from both the displacement of the unstable modes after steepest descent minimization (see Figure 6(a)) and the overall fluctuation change in magnitude after deletion of the $\gamma$-phosphate group (see Figure 9). The coupling, which is likely to involve the displacement of the switch II region, may have functional implications. The recently proposed model of the complex between ras p21 and the GTPase-activating domain of human p120GAP based on the GAP crystal structure indicates that $\alpha 3$ is involved in the interaction (Scheffzek et al., 1996). The decrease of the overall fluctuation of the $\alpha 3$ region upon deletion of the $\gamma$-phosphate group in the G12D mutant (see Figure 9) may affect the binding of GAP.

Comparison of the difference in the behavior of the wild-type and G12D mutant on the removal of the $\gamma$-phosphate group indicates that the latter has a more rigid structure that could slow or inhibit the conformational transition. This suggests that such an oncogenic mutation can affect the conformational transition in a direct fashion, although it appears to be involved also in a decrease in the GTPase activity.

The normal mode results also show an influence of the removal of the $\gamma$-phosphate group on the loop 1 region. This region does not show a significant conformational difference between the GTP and GDP-bound forms. Loop 1 is frequently referred to as the phosphate-binding loop or P-loop (Wittinghofer \& Pai, 1991) and the sequence of this loop is highly conserved in ras p21 across the species. In the wild-type structure, residues of loop 1 do not have direct contact with the $\gamma$-phosphate group, although the loop is spatially close to it. Although the structure is conserved, the vibrational properties of this loop change significantly between the GTP-bound and GDP-bound form, and between the wild-type and the G12D mutant.

The present analysis presents insights into the early stages of the conformational transition between the GTP and GDP-bound forms of ras p21. In subsequent work, the entire transition will be studied to obtain a more complete understanding of the structural changes (Ma \& Karplus, 1997). It is hoped that this will result in information that will be useful for developing drugs that alter the rate or nature of the transition. The results also have more general significance, since there are many other cases where conformational transitions are induced by $\gamma$-phosphate hydrolysis (e.g. in the elongation factor EFTu (Scheffzek et al., 1993)). 


\section{Methods}

\section{Normal mode analysis}

A detailed description of normal mode calculations for macromolecules and how they are implemented in the CHARMM program (Brooks et al., 1983) is given by Brooks et al. (1995). Here, we define some quantities used to interpret the normal modes. First, we introduce the covariance tensor. If $\langle\ldots\rangle$ denotes the ensemble average at a temperature $T$ (McQuarrie, 1976), the covariance tensor, $\mathrm{C}$, of the atomic fluctuation is given by

$$
\begin{gathered}
\mathrm{C} \equiv\left\langle\mathbf{q q}_{\eta}^{\mathrm{T}}\right\rangle \\
=\mu^{-\mathbf{1}} \mathbf{A} \propto \mathbf{A}_{\eta}^{\mathrm{T}} \mu_{\eta}^{-1 \mathrm{~T}}
\end{gathered}
$$

where $\mathbf{q}$ is the Cartesian displacement coordinates of the individual atoms, $\mu$ is defined as $\mathrm{M} \equiv \mu^{T} \eta$ with $\mathrm{M}$ being an atomic mass tensor, A is the unitary transformation matrix between normal modes and their Cartesian projections, matrix $\alpha$ is a diagonal matrix whose diagonal elements are $k_{\mathrm{B}} T / \omega_{i}^{2}$ with $k_{\mathrm{B}}$ the Boltzmann's constant. $T$ is the temperature, $\omega_{i}$ is the frequency of the $i$ th normal mode and the superscript $\mathrm{T}$ indicates a transpose. Both the diagonal and the off-diagonal elements of the covariance matrix are of physical significance. The diagonal elements give the mean-square fluctuation of each Cartesian coordinate at the temperature $T$. The mean-square fluctuations provide information about the flexibility of a protein in the harmonic approximation (Brooks et al., 1988, 1995). They can be related to the thermal $B$-factors measured by X-ray crystallography. Recent work by Go and co-workers have shown the utility of the fluctuations obtained from normal mode calculations for evaluation of the temperature factors in X-ray analysis (Hayward \& Go, 1995). The off-diagonal elements of the matrix C gives the equal-time cross-correlation function between a pair of coordinates. The cross-correlation function is usually expressed in a normalized form so the elements are independent of temperature. Defining a diagonal matrix D with its diagonal elements equal to the inverse square-root of diagonal elements of matrix C, we have:

$$
\mathbf{C}^{\prime}=\mathbf{D}^{\mathrm{T}} \mathbf{C D}
$$

where $\mathbf{C}^{\prime}$ has diagonal elements equal to unity and the off-diagonal elements give the normalized cross-correlation functions, which vary between -1 for anticorrelated motion and +1 for correlated motion. The correlation of the atomic fluctuation is often presented for a pair of atoms rather than a pair of degrees of freedom, i.e. the correlation $\left\langle\Delta \mathbf{r}_{i} \Delta \mathbf{r}_{j}\right\rangle$ is considered so that the original $\mathrm{C}$ matrix must be transformed from a $3 N \times 3 N$ degree-of-freedom-based form to an $N \times N$ atom-based form. If this matrix is denoted by $\mathbf{C}^{\prime \prime}$, then the $i j$ th element of $\mathbf{C}^{\prime \prime}$ is equal to the trace of a corresponding $3 \times 3$ sub-block of the matrix $C$. The transformed matrix $C^{\prime \prime}$ can be normalized in the same way as in equation (3).

A quantity that is of particular utility for the present study is the projection of each normal mode on the direction of a specific conformational change. If $\mathbf{R}_{\mathbf{1}}$ and $\mathbf{R}_{\mathbf{2}}$ denote two distinct conformers of the protein, a unit vector $\mathrm{p}$ :

$$
\mathrm{d} \equiv \frac{\mathbf{R}_{\mathbf{2}}-\mathbf{R}_{\mathbf{1}}}{\left|\mathbf{R}_{\mathbf{2}}-\mathbf{R}_{\mathbf{1}}\right|}
$$

defines the direction of the conformational transition. The projection of the normal modes on the direction of the transition is the inner product of the eigenvector for that mode with the vector $\mathbf{d}$. We have:

$$
\mathbf{I}=\left|\mathbf{A}^{\mathrm{T}} \mathbf{d}\right|
$$

where $\mathbf{I}$ is a $3 N$-dimensional vector with its $i$ th component defined as the "involvement coefficient" of the ith normal mode. The components of I are the absolute values of the inner product, and their values are in the range of $[0,1]$. The involvement coefficient is +1 if a particular normal mode has a vibrational pattern that is parallel with the conformational difference and it is zero if it is orthogonal to the conformational difference (Marques \& Sanejouand, 1995).

\section{Computational procedure}

The normal mode analysis is usually based on the energy-minimized structure of the protein and the motions about that minimum lead to positive eigenvalues and real frequencies. If the system is not at a minimum, negative eigenvalues (imaginary frequencies) can result. For special purposes, as mentioned in the Introduction, they can play a useful role. With the use of the vacuum system and minimization traditional for normal mode analysis (Brooks et al., 1995), the minimized structure can deviate significantly from the original crystal structure, due primarily to the perturbation arising from the charged surface residues. The deviation can be large when a force-field, such as the CHARMM (Brooks et al., 1983) parameter set 22 , that is specifically designed for solvated simulations, is employed in vacuum.

To reduce the structural changes during the energy minimization, a system consisting of the protein plus a shell of water molecules was constructed. A smaller-angle X-ray study (Fujisawa et al., 1994) has estimated that there are about 400 water molecules that are strongly bound to the protein. They correspond to "structural" water molecules that help to stabilize the native structure. In a study on the hydration of myoglobin (Steinbach \& Brooks, 1993), which is a protein of 
similar size to ras p21, it was found that a shell of 350 water molecules yielded structural and dynamic behavior corresponding to that of bulk water. A normal mode analysis with a water shell was made for crambin earlier (Teeter \& Case, 1990).

We used the CHARMM program (Brooks et al., 1983) and the all-hydrogen potential function (PARAM22: Mackerell et al., 1997). For each crystal structure studied, we created a water solvation shell with about 400 water molecules. The original crystal water molecules, if any, were kept and additional water molecules were added by overlapping the crystal structure with a previously prepared, large equilibrated water box (at $300 \mathrm{~K}$ ) and deleting all water molecules located within a distance of $2.8 \AA$ of a protein atom or crystal water molecules. The final number of water molecules was adjusted to be around 400 . The actual number of water molecules varies between 395 and 405 for the different crystal structures. To be consistent with the normal mode calculation, a fully flexible TIP3P water model (Dang \& Pettitt, 1987) was used.

A 50 ps equilibration dynamics run at $300 \mathrm{~K}$ was made with the rigid protein to relax the solvent molecules. The ligand waters of the $\mathrm{Mg}^{2+}$ in the active site were also kept fixed. The leap-frog method (Allen \& Tildesley, 1980) in CHARMM was used as the numerical integrator for the equations of motion. A $0.5 \mathrm{fs}$ time-step was used because of the high-frequency vibrational motions of the bonds involving hydrogen atoms; the SHAKE algorithm was not employed. The nonbonded electrostatic interaction was shifted to zero at $12 \AA$ and the van der Waals interaction was switched off from $10 \AA$ (Brooks et al., 1988). The corresponding cutoff distance for the nonbonded neighbor list update was $13 \AA$. The nonbonded neighbor list was updated by the heuristic method in the CHARMM program, i.e. only when the largest displacement of an atom in the system exceeded half of the thickness $(0.5 \AA)$ of the outer neighbor list shell. The initial velocities were assigned by randomly sampling a Maxwellian distribution at $300 \mathrm{~K}$. The water shell was slowly heated from 0 to $300 \mathrm{~K}$ during the first 5 ps simulation with a temperature increment of $6 \mathrm{~K}$ every $0.1 \mathrm{ps}$. For the rest of the $45 \mathrm{ps}$ simulation, the temperature was scaled if the fluctuation exceeded $300( \pm 5) \mathrm{K}$. The final equilibrated solvent structure together with the protein was energy minimized by 2000 steps with the steepest descent method and then 5000 steps with the Adapted Basis Newton Raphson method in CHARMM to obtain a well-minimized structure. Harmonic constraints were used during the minimization and the strength of the constraints was slowly decreased to zero. The final rms gradient was $10^{-7} \mathrm{kcal} / \mathrm{mol} \AA$. Previous studies (Tidor \& Karplus, 1994) have shown that such a rms gradient is satisfactory for calculating normal modes; it is expected to yield only real frequencies. The force constant matrix including all the degrees of freedom was created by the VIBRAN module of the CHARMM program and diagonalized to determine the normal modes and normal mode frequencies.

In addition to determining the normal modes for the minimized crystal structure, we calculated the normal modes of the structure with the $\gamma$-phosphate group removed to examine the origins of the conformational transitions upon the hydrolysis of the $\gamma$-phosphate group. The structure was obtained by deleting the $\gamma$-phosphate group from the minimized GTP-bound structure. The atomic charges on the GDP fragment were adjusted to be the same as that of normal GDP.

Five different structures were used. They are the wild-type GDP-bound form (Protein Data Bank (PDB) code 1q21; Tong et al., 1991), the wild-type GTP-bound form (PDB code 5p21; Pai et al., 1990), the same structure with the $\gamma$-phosphate group removed (denoted as the GTP* form) and the GTP-bound G12D mutant (PDB code lagp; Franken et al., 1993) and the same structure with the $\gamma$-phosphate group removed (denoted as the G12D* form). The crystal structures of the GTP-bound form of the wild-type and the G12D mutant contain a non-hydrolyzable GTP analog. This GTP analog is guanosine $5^{\prime}-(\beta, \gamma-$ imido) triphosphate (GppNHp); in the simulations, a model of the substrate GTP in the active site was used. Also, one of the $\mathrm{O}$ atoms of the $\gamma$-phosphate group of the G12D mutant appeared to be protonated in the crystal structure (Franken et al., 1993); we adopted this protonation state for the calculation. The extra hydrogen atom makes an important hydrogen bonding contact with an oxygen atom of the side-chain of Asp12.

\section{Acknowledgments}

This work was supported in part by a grant from National Institute of Health. We thank Dr Carla Mattos for helpful discussions. J. M. is a Burroughs Wellcome PMMB Postdoctoral fellow.

\section{References}

Allen, M. P. \& Tildesley, D. J. (1980). Computer Simulation of Liquids. Clarendon Press, Oxford.

Barbacid, M. (1987). ras Genes. Annu. Rev. Biochem. 56, 779-827.

Brooks, B. \& Karplus, M. (1985). Normal mode for specific motions of macromolecules: application to the hinge-bending mode of lysozyme. Proc. Natl Acad. Sci. USA, 82, 4995-4999.

Brooks, B. R., Bruccoleri, R. E., Olafson, B. D., States, D. J., Swaminathan, S. \& Karplus, M. (1983). CHARMM: a program for macromolecular energy, minimization, and dynamics calculations. J. Comput. Chem. 4, 187-217. 
Brooks, C. L., Karplus, M. \& Pettitt, B. M. (1988). Proteins: a theoretical perspective of dynamics, structure, and thermodynamics. Advan. Chem. Phys. 71, $1-249$.

Brooks, B. R., Janežič, D. \& Karplus, M. (1995). Harmonic analysis of large systems. I. Methodology. J. Comput. Chem. 16, 1522-1542.

Choi, J. K. \& Straub, J. E. (1994). Extracting the energy barrier distribution of a disordered system from the instantaneous normal mode density of states: applications to peptides and proteins. J. Phys. Chem. 98, 10978-10987.

Dang, L. X. \& Pettitt, B. M. (1987). Simple intramolecular model potentials for water. J. Phys. Chem. 91, 3349-3354.

Der, C. J., Finkel, T. \& Cooper, G. M. (1986). Biological and biochemical properties of human $\mathrm{ras}^{\mathrm{H}}$ genes mutated at codon 61. Cell, 44, 167-176.

Díaz, J. F., Wroblowski, B. \& Engleborhs, Y. (1995). Molecular dynamics simulation of the solution structures of Ha-ras-p21 gdp and gtp complexes: flexibility, possible hinges, and levers of the conformational transition. Biochemistry, 34, 12038-12047.

Feig, L. A. (1994). Guanine-nucleotide exchange factors: a family of positive regulators of Ras and related GTPases. Curr. Opin. Cell Biol. 6, 204-211.

Foley, C. K., Pedersen, L. G., Charifson, P. S., Darden, T. A., Wittinghofer, A., Pai, E. F. \& Anderson, M. W. (1992). Simulation of the solution structure of the H-ras p21-GTP complex. Biochemistry, 31, 4951-4959.

Franken, S. M., Scheidig, A. J., Krengel, U., Renslan, H., Lautwein, A., Geyer, M., Scheffzek, K., Good, R. S., Kalbitzer, H. R., Pai, E. F. \& Wittinghofer, A. (1993). Three-dimensional structures and properties of a transforming and a nontransforming Gly-12 mutant of p21 ${ }^{\mathrm{H}-\mathrm{ras}}$. Biochemistry, 32, 8411-8420.

Fujisawa, T., Uraga, T., Yamaizumi, Z., Inoko, Y., Nishimura, S. \& Ueki, T. (1994). The hydration of ras p21 in solution during GTP hydrolysis based on solution X-ray scattering profile. J. Biochem. 115, 875-880.

Gibbs, J. B., Sigal, I. S., Poe, M. \& Scolnick, E. M. (1984). Intrinsic GTPase activity distinguishes normal and oncogenic ras p21 molecules. Proc. Natl Acad. Sci. USA, 81, 5704-5708.

Harrison, R. W. (1984). Variational calculation of the normal modes of a large macromolecule: methods and some initial results. Biopolymers, 23, 2943-2949.

Hayward, S. \& Go, N. (1995). Collective variable description of native protein dynamics. Annu. Rev. Phys. Chem. 46, 223-250.

Howe, L. R. \& Marshell, C. J. (1993). Identification of amino acids in p21ras involved in exchange factor interaction. Oncogenes, 8, 2583-2590.

Joseph, D., Petsko, G. A. \& Karplus, M. (1990). Anatomy of a conformational change: hinged "lid" motion of the trioseposphate isomerase loop. Science, 249, $1425-1428$.

Kjeldgaard, M., Nyborg, J. \& Clark, B. F. (1996). The GTP binding motif: variations on a theme. FASEB J. 10, 1347-1368.

Kraulis, P. J. (1991). MOLSCRIPT: a program to produce both detailed and schematic plots of protein structures. J. Appl. Crystallog. 24, 946-950.

Kuriyan, J. \& Weis, W. (1991). Rigid protein motion as a model for crystallographic temperature factors. Proc Natl Acad. Sci. USA, 88, 2773-2777.
Lowy, D. R. \& Willumsen, B. M. (1993). Function and regulation of ras. Annu. Rev. Biochem. 62, 851-891.

Ma \& Karplus (1997), NAS, in press.

Mackerell (1997). J. Phy. Chem., in press.

Manne, V., Bekesi, E. \& Kung, H. (1985). Ha-ras proteins exhibit GTPase activity: point mutations that activate ha-ras gene products result in decreased GTPase activity. Proc. Natl Acad. Sci. USA, 82, 376380.

Marques, O. \& Sanejouand, Y. H. (1995). Hinge-bending motion in citrate synthase arising from normal mode calculations. Proteins: Struct. Funct. Genet. 23, 557-560.

McCammon, J. A., Gelin, B. R. \& Karplus, M. (1977). Dynamics of folded proteins. Nature, 267, 585590.

McCormick, F. (1989). ras GTPase activating protein: signal transmitter and signal terminator. Cell, 56, 5-8.

McQuarrie, D. A. (1976). Statistical Mechanics. HarperCollins, New York.

Milburn, M. V., Tong, L., deVos, A. M., Brünger, A., Yamaizumi, Z., Nishimura, S. \& Kim, S. H. (1990). Molecular switch for signal transduction: structural differences between active and inactive forms of protooncogenic ras p21. Science, 247, 939-945.

Neal, S. E., Eccleston, J. F. \& Webb, M. R. (1990). Hydrolysis of GTP by p21 $1^{\text {NRAS }}$ protooncogene product, is accompanied by a conformational change in the wild-type protein: use of a single fluorescent probe at the catalytic site. Proc. Natl Acad. Sci. USA, 87, 3562-3565.

Pai, E. F., Krengel, U., Petsko, G. A., Goody, R. S., Kabsch, W. \& Wittinghofer, A. (1990). Refined crystal structure of the triphosphate conformation of H-ras p21 at 1.35 å resolution: implications for the mechanism of GTP hydrolysis. EMBO J. 9, 23512359.

Sanejouand, Y. H. (1996). Normal-mode analysis suggests important flexibility between the two $\mathrm{N}$-terminal domains of CD4 and supports the hypothesis of a conformational change in CD4 upon HIV binding. Protein Eng. 9, 671-677.

Scheffzek, K., Lautwein, A., Kabsch, W., Ahmadian, M. R. \& Wittinghofer, A. (1993). The crystal structure of elongation factor EF-Tu from thermus acquaticus in the GTP conformation. Structure, 1, 35-50.

Scheffzek, K., Lautwein, A., Kabsch, W., Ahmadian, M. R. \& Wittinghofer, A. (1996). Crystal structure of the GTPase-activating domain of human p120GAP and implications for the interaction with Ras. Nature, 384, 591-596.

Seeburg, P. H., Colby, W. W., Capon, D. J., Goeddel, D. V. \& Levinson, A. D. (1984). Biological properties of human c-Has-ras 1 genes mutated at codon 12 . Nature, 312, 71-75.

Steinbach, P. J. \& Brooks, B. (1993). Protein hydration elucidated by molecular dynamics simulation. Proc. Natl Acad. Sci. USA, 90, 9135-9139.

Stratt, R. M. (1995). The instantaneous normal modes of liquids. Acc. Chem. Res. 28, 201-207.

Teeter, M. M. \& Case, D. A. (1990). Harmonic and quasiharmonic description of crambin. J. Phys. Chem. 94, 8091-8097.

Thomas, A., Field, M. J., Mouawad, L. \& Perahia, D. (1996a). Analysis of the low frequency normal modes of the T-state of aspartate transcarbamylase. J. Mol. Biol. 257, 1070-1087. 
Thomas, A., Field, M. J. \& Perahia, D. (1996b). Analysis of the low frequency normal modes of the $\mathrm{R}$ state of aspartate transcarbamylaseand a comparison with the T state modes. J. Mol. Biol. 261, 490-506.

Tidor, B. \& Karplus, M. (1994). The contribution of vibrational entropy to molecular association. J. Mol. Biol. 238, 405-414.
Tong, L., de Vos, A. M., Milburn, M. V. \& Kim, S.-H. (1991). Crystal structures at $2.2 \AA$ resolution of the catalytic domains of normal ras protein and an oncogenic mutant complexed with GDP. J. Mol. Biol. 217, 503-516.

Wittinghofer, A. \& Pai, E. (1991). The structure of ras protein: a model for a universal molecular switch. Trends Biochem. Sci. 10, 382-387.

Edited by I. B. Honig

(Received 27 January 1997; received in revised form 4 August 1997; accepted 4 August 1997) 